\section{An Automated Chamber System for Measurements of Whole-vine Gas Exchange}

\author{
S. Poni, E. Magnanini, and B. Rebucci \\ Dipartimento di Colture Arboree, Sezione Viticola Centro Ricerche Viticole ed \\ Enologiche, Università di Bologna, Via Filippo Re,6, 40126 Bologna, Italy
}

Additional index words. photosynthesis, grapevine, transpiration

\begin{abstract}
The reported system interfaces a commercially available portable infrared gas analyzer with a measurement and control module for continuous and automated measurements of whole-canopy gas exchange. Readings were taken for several days, under mostly sunny or partly cloudy conditions, on two potted vines (total leaf area per vine of $\approx 1.3 \mathrm{~m}^{2}$ ) enclosed in inflated polyethylene chambers. The air flow rate through the chambers was provided by a centrifugal blower and set at $5 \mathrm{~L} \cdot \mathrm{s}^{-1}$ by a butterfly valve. It prevented $\Delta \mathrm{CO}_{2}$ from dropping below $-40 \mathrm{~mL} \cdot \mathrm{L}^{-1}$. Switching of the two $\mathrm{CO}_{2}$ analysis channels to the infrared gas analyzer (operated in a differential mode) was achieved by solenoid valves, whereas wet and dry-bulb temperatures at chambers' inlet and outlet were measured by low-cost, custom-made thermocouple psychrometers. Whole-vine assimilation rate (WVA) and whole-vine transpiration rate were calculated from the inlet-outlet differences in $\mathrm{CO}_{2}$ and absolute humidity. When compared to assimilation measured on single leaves (SLA) under saturating light at equivalent times, the WVA reduction (area basis) was $\approx \mathbf{5 0 \%}$, suggesting that whole-canopy photosynthetic efficiency based on SLA readings can be greatly overestimated.
\end{abstract}

Whole-canopy gas exchange can be estimated by using enclosure or micrometeorological methods (flux-gradient or eddy correlation) based on analyses of fluxes through crop boundary layers (Garcia et al., 1990; Mitchell, 1992). Canopy discontinuity of fruittree plantings and the need of large areas of homogeneous vegetation make the micrometeorological approach problematic (McMillen, 1988). Enclosure methods seem to have such advantages as 1) more accurate estimates of whole-plant gas exchange; 2) good adaptation to small plot size, thereby making easier the comparison of several treatments; 3) lower cost than the micrometeorological systems; and 4) overcoming the inherent limitations of portable, single-leaf gas-exchange units.

The use of large chambers to enclose a tree's whole-shoot system for specific gasexchange measurements has been reconsidered several decades after Heinicke and Childers (1936) conceived and built an assimilation chamber to study a whole apple tree's gas exchange in the field. The interest today seems to be restricted to closed and open passive systems, which have distinct advantages in terms of simplicity, cost, and transportability with respect to controlled-environment, closed-chamber systems or compensating open-chamber systems. While the preference for the open or closed whole-plant cham-

Received for publication 28 Mar. 1996. Accepted for publication 9 Aug. 1996. Research supported by the National Research Council of Italy, Special Project RAISA, subproject no. 2, paper no. 2810. The cost of publishing this paper was defrayed in part by the payment of page charges. Under postal regulations, this paper therefore must be hereby marked advertisement solely to indicate this fact. ber still is being debated, several recent contributions (Buwalda et al., 1992; CorelliGrappadelli and Magnanini, 1993; Edson et al., 1993; Katerjii et al., 1994; Lakso et al., 1994; Succi et al., 1990) stress the disadvantages of the closed (transient) systems (measurements only reliable over a short time period, high sensitivity to leakiness, etc.) and the advantages of open-chamber ones. Among the latter systems, that of Corelli-Grappadelli and Magnanini (1993) reduced the bulk and cost of the whole apparatus.

The present study reports further improvements of this system aimed to automate gas sampling without significantly increasing the complexity of the system or compromising its flexibility (single-leaf vs. whole-tree). Measurements were taken on two isolated potted vines as examples of the data that the system can deliver. Whole-canopy photosynthetic rates also were compared to photosynthetic rates recorded by a standard single-leaf portable unit.

\section{Materials and Methods}

Gas-exchange apparatus. The system(Fig. 1), similar in design to that of CorelliGrappadelli and Magnanini (1993), included an alternating-current (AC), air-feed centrifugal blower (C20/2M Vortice, Milan, Italy) for a maximum flow rate of $\approx 267 \mathrm{~L} \cdot \mathrm{s}^{-1}$; two flexible, inflated, 0.025-mm-thick, transparent polyethylene chambers $(210 \mathrm{~cm}$ high, $120 \mathrm{~cm}$ wide); a portable infrared gas analyzer (LCA1, Analytical Development Co., Hoddesdon, U.K.); and a CR10 datalogger and control module (Campbell Scientific, Leicestershire, U.K.) for system programming and data storing and processing.
Inlet air was drawn from $\approx 4 \mathrm{~m}$ above the soil surface and directed by the blower through a 100-mm-diameter, rigid plastic pipe. The air flow was split and diverted to two chambers via a flexible, internally smoothed PVC pipe (45 $\mathrm{mm}$ in diameter). Flow to each chamber was controlled by a butterfly valve.

The polyethylene chambers were secured at the top to a support stake and at the bottom to the trunk, the stake, and the flexible pipe. Paper or foam-rubber gasket materials were used to seal the gaps. Low-cost, custom-made thermocouple psychrometers (Fig. 2) were used to measure wet- and dry-bulb temperatures at the chamber's inlet and outlet. To allow proper ventilation, the first psychrometer was fitted by an open-ended $\mathrm{T}$ to the pipe segment preceding the shunt, while the psychrometers at the chamber outlets were connected to a tapered pipe segment atop each stake.

Dry-bulb temperatures $\left(t_{d}\right)$ were used to calculate the saturation vapor pressure over water $\left(\mathrm{e}_{\mathrm{s}}\right)$ according to Lowe and Paul (1977). Inlet and outlet vapor pressures (e) were computed from dry- and wet-bulb temperatures $\left(t_{w}\right)$ by the algorithm of the U.S. National Weather Service (function 57 in the processing instructions of the CR10 manual). Relative humidities $(\mathrm{RH})$ were then calculated as $\mathrm{e} / \mathrm{e}_{\mathrm{s}(\mathrm{d})}$ and the absolute humidities (Hs) were given by the equation reported by the American Society of Agricultural Engineers (1982). Ambient temperature $\left(t_{2}\right)$ was monitored continuously by a 0.51 -mm-diameter, PVC-insulated, copper-constantan thermocouple placed at 1.8 $\mathrm{m}$ from the ground inside a meteorological shelter and connected to the CR10 module for 10 -min recording intervals.

The LCA-1 infrared gas analyzer was operated in differential mode to continuously compare the reference $\mathrm{CO}_{2}$ drawn from the inlet psychrometer location and the two $\mathrm{CO}_{2}$ analysis streams derived from the abovecanopy psychrometer outlets. Switching of the two $\mathrm{CO}_{2}$ analysis channels to the LCA-1 was achieved by a solenoid valve $(12 \mathrm{~V}-300$ $\mathrm{mA}$, usually closed; SIRAI, Milan, Italy) set between the two $\mathrm{CO}_{2}$ lines and the analysis inlet on the IRGA. The control program had an 80 -s switching interval; the initial $20 \mathrm{~s}$ was for flushing, and readings taken at 40,60, and 80 $\mathrm{s}$ were averaged and stored. Two columns of silica-gel were connected by plastic adaptors to the reference and analysis inlets of the LCA1 to minimize cross-sensitivity to water vapor. Due to the similar amount of leaf area enclosed in the chambers, the flow was adjusted for both to $\approx 5 \mathrm{~L} \cdot \mathrm{s}^{-1}$, resulting in a $\mathrm{CO}_{2}$ differential never $>40 \mu \mathrm{mol} \cdot \mathrm{mol}^{-1}$. The air flow through each chamber was assessed at the beginning of measurements and then checked repeatedly by determining the time needed to fill a polyethylene, custom-made cylindrical bag having a volume of $920 \mathrm{~L}$. Since the volume of the chambers was estimated at $\approx 500 \mathrm{~L}$, a complete volume change occurre every $100 \mathrm{~s}$. To verify potential error due to back-pressure during bag inflation, a comparison was made to the flow restriction method of Osborne (1977). At reference air speeds of $4,4.8$, and $5.8 \mathrm{~L} \cdot \mathrm{s}^{-1}$, as 

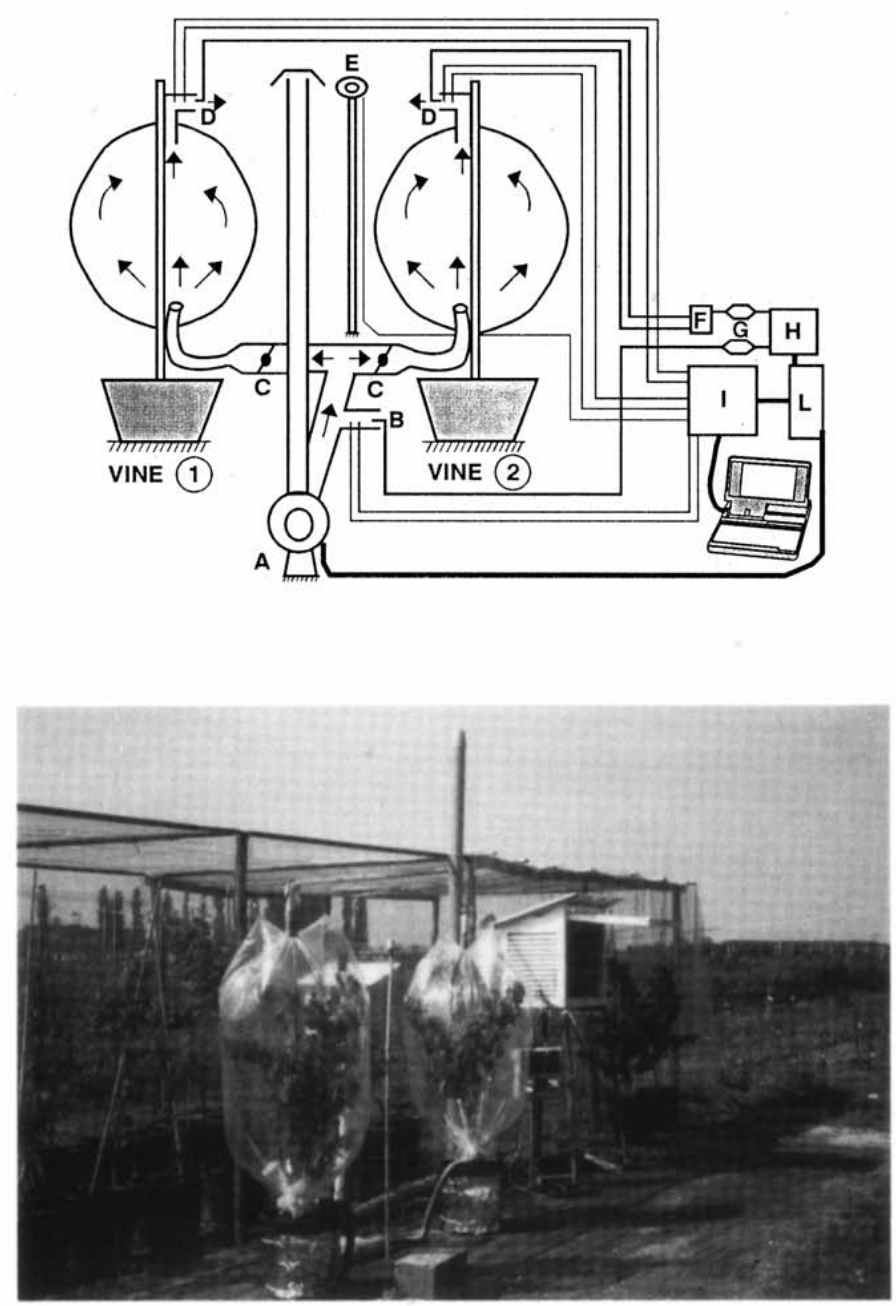

Fig.1. (top) Schematic and (bottom) actual configuration of the automated chamber system. Main components are: (A) AC blower; (B) chamber inlet for measuring reference $\mathrm{CO}_{2}$, dry- and wet-bulb temperatures; (C) butterfly valves regulating flow; (D) chamber outlets for measuring $\mathrm{CO}_{2}$ and dry-and wet-bulb temperatures; (E) cosine-corrected quantum sensor; (F) solenoid (normally closed); (G) silicagel columns; (H) ADC-LCA-1 infrared gas-analyzer; (I) Campbell CR10 measurement and control module; and (L) power pack.

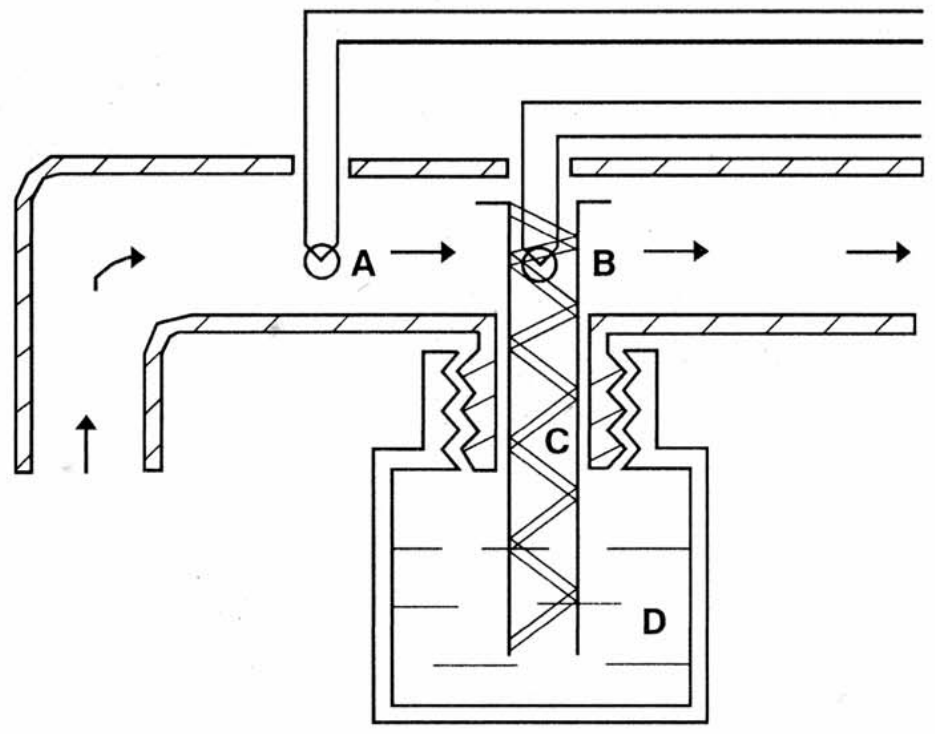

Fig. 2. Diagram of the home-built thermocouple psychrometer. A curved segment of white plastic pipe (40 $\mathrm{mm}$ in diameter) housed an upstream ventilated dry junction (A) and a downstream ventilated wet junction (B). Wetting of the thermocouple junction was assured by a cotton string that remained in a container (D) of distilled water. Before use, the string was washed with ethanol to increase absorbance. determined by flow restriction, the inflation method resulted in 4.1, 5.1, and $6.5 \mathrm{~L} \cdot \mathrm{s}^{-1}(r=$ 0.99 ), respectively, indicating errors $<9 \%$ and good reliability across the set operating flow of $5 \mathrm{~L} \cdot \mathrm{s}^{-1}$.

Incident photosynthetically active radiation (PAR) was monitored continuously by a cosine-corrected Silimet RG39 quantum sensor (Silimet, Modena, Italy) placed horizontally on top of a support stake next to the chambers. The quantum sensor was wired to the CR10 module for storage and transformation of the output voltage to $\mu \mathrm{mol} \cdot \mathrm{m}^{-2} \cdot \mathrm{s}^{-1}$ (1 $\mathrm{mV}=40 \mu \mathrm{mol} \cdot \mathrm{m}^{-2} \cdot \mathrm{s}^{-1}$. The low-density polyethylene film (M.O.P., Bologna, Italy) enclosing the vine is known not to alter the spectral composition of light while the percentage of transmission is decreased by a maximum of $12 \%$ within the visible range (CorelliGrappadelli and Magnanini, 1993).

CR10 programming and data manipulation were performed by the PC208 Datalogger Support Software (Campbell Scientific). Under the current configuration, the control program downloaded to the CR 10 provided a string of 17 elementary or partially processed parameters [program code, day of year, time ( $h, \min$ ), time (s), PAR, $t_{d}$ inlet, $t_{d}$ outlet $1, t_{d}$ outlet $2, \mathrm{t}_{\mathrm{w}}$ inlet, $\mathrm{t}_{\mathrm{w}}$ outlet $1, \mathrm{t}_{\mathrm{w}}$ outlet $2, \mathrm{CO}_{2}$ reference, $\mathrm{CO}_{2}$ analysis, code for chamberidentification, $\mathrm{H}$ inlet, $\mathrm{H}$ outlet $1, \mathrm{H}$ outlet 2] automatically stored every $80 \mathrm{~s}$. The CR 10 wiring panel allowing the above configuration is represented in Fig. 3. Unloading of the stored data or periodical checks of overall system functioning was performed by a portable PC connected to the CR 10 module by a SC32A, an optically isolated RS232 interface.

Plantmaterial.Four-year-old, well-watered, own-rooted, fruiting 'Chardonnay' grape (Vitis vinifera $\mathrm{L}$.) vines grown outside in $45-\mathrm{L}$ pots containing a medium of 2.5 peat : 1.5 sand : 1 soil (v/v) were used for initial tests. Two vines were selected for uniformity of shoot count $(\approx 10$ to 12 per vine $)$ and thinned to one cluster per shoot. In early July, canopies were bushlike, resembling, on a smaller scale, the geometry usually found on goblet-trained vines in the field. This shape was induced deliberately to minimize the diurnal variability resulting from canopy orientation with respect to the solar track. The sides of the pots were wrapped with aluminum foil to control radiation-induced heating of the root zone. Pot water capacity, gravimetrically estimated at $\approx 11 \mathrm{~L}$, largely exceeded daily total vine loss of $\mathrm{H}_{2} \mathrm{O}$ calculated from transpiration rates, yielding an average of $2 \mu \mathrm{mol} \cdot \mathrm{m}^{-2} \cdot \mathrm{s}^{-1}$ over the light period, or an estimated daily loss of $1.9 \mathrm{~L}$. Pest control prevented significant damage to the leaves.

Gas-exchange measurements. Automated whole-vine gas-exchange measurements started on 13 July and continued for several days. There was no canopy growth throughout the experimental period. On $18 \mathrm{July}$, the chambers were removed temporarily to allow singleleaf assimilation (SLA) readings designed to estimate maximum photosynthetic rates. These were performed from 1030 to $1100 \mathrm{HR}$ and from 1330 to $1400 \mathrm{HR}$ on 15 well-exposed, 


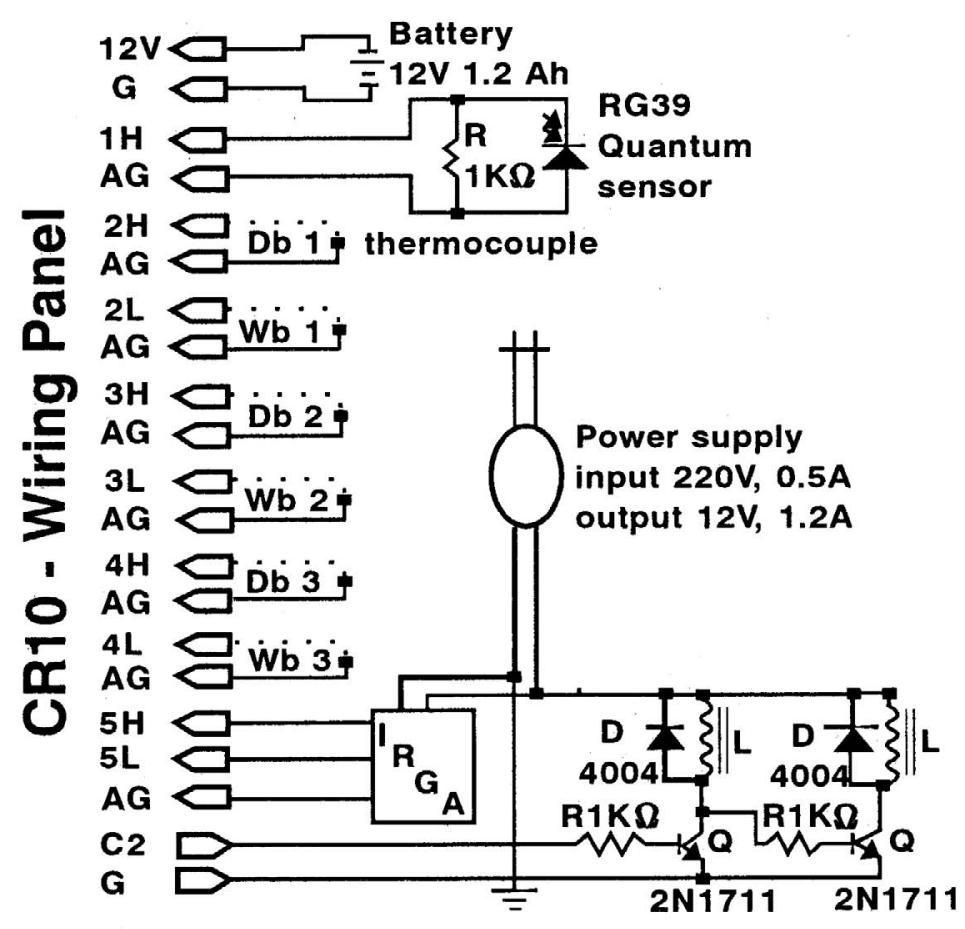

Fig. 3. Configuration of the CR10 wiring panel for the automated whole-vine gas-exchange system. It used nine single-ended analogue input terminals for output voltage of PAR $(1 \mathrm{H}), \mathrm{t}_{\mathrm{d}}(2 \mathrm{H}, 3 \mathrm{H}, 4 \mathrm{H}), \mathrm{t}_{\mathrm{w}}(2 \mathrm{~L}, 3 \mathrm{~L}$, $4 \mathrm{~L}$ ), reference, and analysis $\mathrm{CO}_{2}(5 \mathrm{H}$ and $5 \mathrm{~L})$. One digital input-output port ( $\left.\mathrm{C} 2\right)$ allowed on/off control of the solenoid. AG and G represent analog and power ground references, respectively. This CR10 setting filled 10 of the 12 channels available under the standard configuration, making unnecessary the need for multiplexing. $\mathrm{D}_{\mathrm{b}}=$ dry bulb; $\mathrm{W}_{\mathrm{b}}=$ wet bulb; $\mathrm{R}=$ resistor; $\mathrm{D}=$ diode; $\mathrm{Q}=$ transistor; $\mathrm{L}=$ solenoid valve.
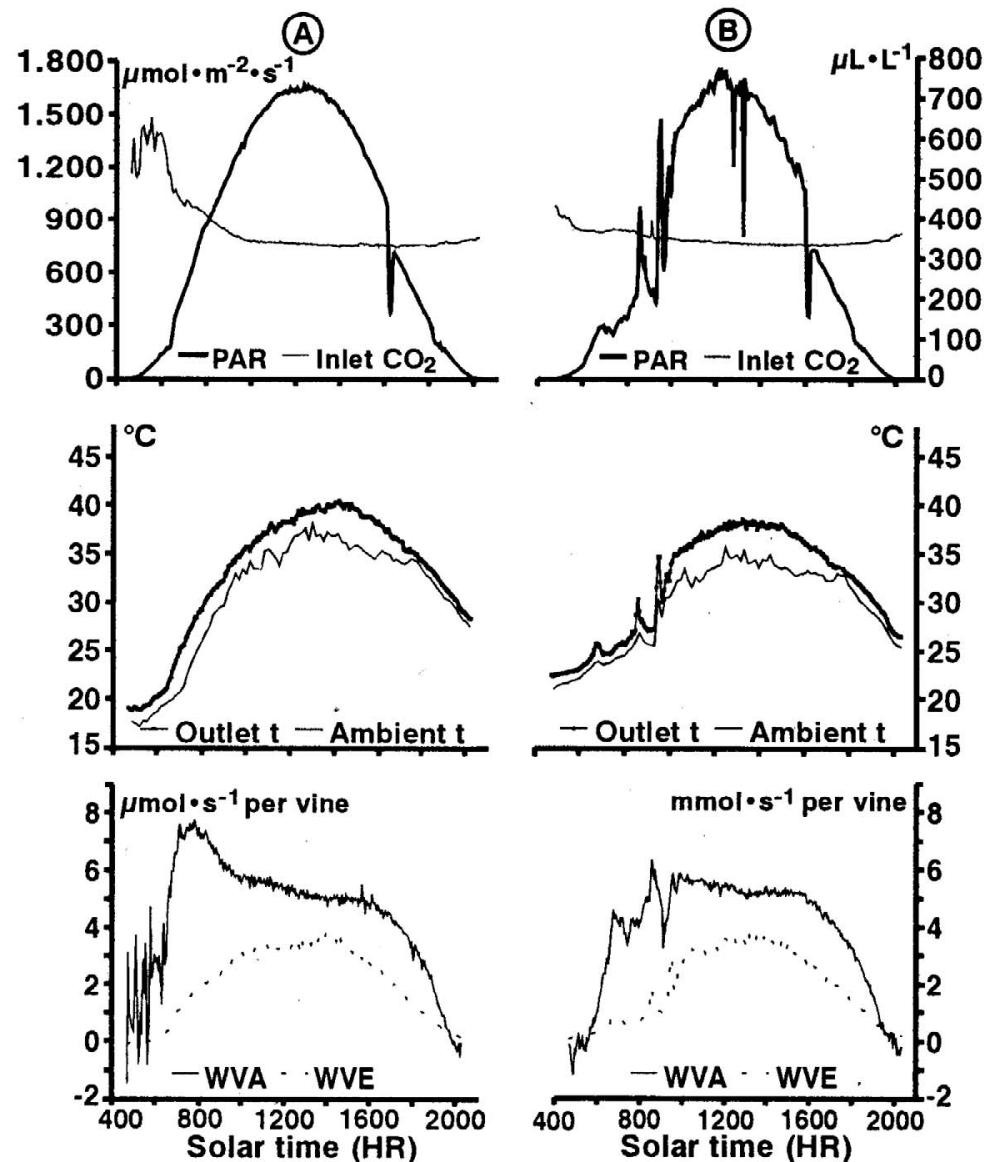

Fig. 4. Trends in PAR, inlet $\mathrm{CO}_{2}$, outlet temperature, ambient temperature, WVA, and WVE averaged over the two vines for a clear day (17 July, A) and a partially cloudy day (15 July, B). mid-shoot leaves per vine using the same LCA1-ADC portable infrared gas analyzer fitted with a Parkinson broad leaf chamber $\left(6.25 \mathrm{~cm}^{2}\right)$ at an air flow of $400 \mathrm{~mL} \cdot \mathrm{min}^{-1}$.

Upon completion of the gas-exchange measurements (end of July), all leaves were stripped from each test vine, and the area of each leaf determined by a LI-COR 3000 leaf area meter (LI-COR, Lincoln, Neb.). Wholevine assimilation rate (WVA) and whole-vine transpiration rate (WVE) were calculated from the inlet-outlet differences in $\mathrm{CO}_{2}$ and absolute humidity. At harvest, yield per vine was recorded as well and crop load expressed as the leaf : fruit ratio $\left(\mathrm{cm}^{2} \cdot \mathrm{g}^{-1}\right)$.

\section{Results and Discussion}

Vines 1 and 2 reached similar total leaf areas (1.26 and $1.34 \mathrm{~m}^{2}$, respectively) and yields (1.03 and $1.12 \mathrm{~kg}$, respectively). The resulting crop loads were 12.3 and $11.9 \mathrm{~cm}^{2} \cdot \mathrm{g}^{-1}$, respectively, which indicate adequate source availability (Kliewer and Weaver, 1971; Poni et al., 1993)

Measurement of canopy gas-exchange. Diurnal trends obtained by the automated system from dawn to dusk of environmental parameters representative of a mostly sunny day (17 July) are reported as means of the two vines along with the calculated WVA and WVE per vine (Fig. 4A). For comparison, a corresponding set of results for a partially cloudy day (15 July) is also included (Fig. 4B). The ambient $\mathrm{CO}_{2}$ concentration showed a generally strong variation over the $24 \mathrm{~h}$ ranging from a constant value of $\approx 330$ to 335 $\mu \mathrm{mol} \cdot \mathrm{mol}^{-1}$ during the day, from 0900 to 2000 $\mathrm{HR}$, to as high as $650 \mu \mathrm{mol} \cdot \mathrm{mol}^{-1}$ before dawn (Figs. 4A and B). A similar degree of variability was observed regardless of measurement day and likely can be explained by the fact that the test vines were located among potted fruit trees. The amplitude of day-to-day ambient $\mathrm{CO}_{2}$ variation likely reflects specific wind speed, with larger variations expected under conditions of still air. Maximum PAR, never exceeding $1800 \mu \mathrm{mol} \cdot \mathrm{m}^{-2} \cdot \mathrm{s}^{-1}$, was reached between 1200 and $1400 \mathrm{HR}$.

The temperature gradient from inside the chamber to the outside was kept within 2 to 4.5 ${ }^{\circ} \mathrm{C}$ over the test periods (Fig. $4 \mathrm{~A}$ and B). The maximum ambient temperatures over the test days (rising to $37^{\circ} \mathrm{C}$ ) are typical of the summer climate in central Po Valley.

On 17 July, WVA peaked for both vines at $0800 \mathrm{HR}$ before declining rapidly until $\approx 1000$ HR (Fig. 4A). It then stayed essentially constant or moderately decreased until the more rapid decline associated with a lowering irradiance. A similar trend also was found for the more cloudy day (Fig. 4B), when maximum WVA occurred in early morning despite the light fluctuations. On the same day, canopy photosynthesis showed rapid adjustments to variable PAR.

The net WVA decline recorded at midmorning seems to be related to the progressive depletion of ambient $\mathrm{CO}_{2}$ until an equilibrium level was reached. This result is in close agreement with the pattern found during whole- 
vine gas-exchange measurements of mature kiwifruit vines [A. deliciosa (A. Chev.) C.F. Liang et A.R. Ferguson var. deliciosa] in the field by Buwalda et al. (1992). This suggests caution when trying to extrapolate single-leaf photosynthesis data to the whole canopy, especially if the leaf analysis system controls $\mathrm{CO}_{2}$ concentration.

The diurnal trend of WVE of both vines closely followed PAR and cuvette temperature, reaching a maximum during the central hours of the day (Fig. 4 A and B). The diurnal plots of inlet $\mathrm{H}$ (not shown) confirm that this parameter is quite stable despite large changes in the magnitude of its components; however, the fact that inlet and outlet $\mathrm{H}$ constantly tended to overlap under conditions of low light represents a valid baseline from which to start for transpiration estimates.

Comparison of WVA/LA and SLA. The whole-canopy averaged leaf assimilation rates (WVA/LA) recorded on 17 July were $\approx 50 \%$ of the exposed, single-leaf assimilation (SLA) taken the next day at equivalent time-windows (Table 1).

Due to the objective complexity of determining accurately the total leaf area of entire trees, direct comparison of WVA/LA and SLA is reported rarely. In agreement with our study, Edson et al. (1993) concluded that SLA may overestimate WVA/LA even in the case of very small vines (final leaf area varying from 4000 to $7200 \mathrm{~cm}^{2}$ ), which should be characterized by a minimum level of internal shading. Although WVA estimates inherently include the gas-exchange contribution of nonphotosynthesizing and respiring organs like cordons, canes, and clusters, the larger differences found here probably correlate with the complexity of leaf exposure in the canopies. Some leaves are shaded and a fraction of the leaves at the exterior of the canopy is invariably at a sharp angle to the solar direction, thereby receiving nonsaturating radiation. The difference between WVA/LA and SLA would be expected to widen further in high-vigor canopies where a large fraction of leaf area can become heavily shaded by the external leaf layers. Additionally, WVA/LA represents the mean activity of the entire leaf community, including young, actively growing leaves and basal, senescing ones that, in grapevines, typically show lower photosynthetic rates than mature, mid-shoot leaves (Intrieri et al., 1992; Kriedemann et al., 1970; Poni et al., 1994). If these sources of variation are not taken into account during single-leaf measurements by choosing leaves at several positions along the stem (as deliberately avoided in this study), SLA can be biased further towards an overestimate of the true whole-canopy rates.

Thus, once it has been ascertained that the chamber microclimate is unlikely to place significant limitations on the canopy gasexchange, the direct comparison of WVA/LA and SLA can provide a useful index to quan-

Table 1. Comparison of single-leaf assimilation rate (SLA) vs. whole-vine averaged leaf assimilation rate (WVA/LA) taken during two consecutive sunny days at equivalent time windows. ${ }^{2}$

\begin{tabular}{|c|c|c|c|c|c|}
\hline \multirow{4}{*}{$\begin{array}{l}\text { Experimental } \\
\text { vine }\end{array}$} & \multirow{4}{*}{$\begin{array}{c}\text { Leaf area } \\
\left(\mathrm{m}^{2}\right)\end{array}$} & \multicolumn{4}{|c|}{ Assimilation rate $\left(\mu \mathrm{mol} \cdot \mathrm{m}^{-2} \cdot \mathrm{s}^{-1}\right)$} \\
\hline & & \multicolumn{2}{|c|}{ SLA $^{y}$} & \multicolumn{2}{|c|}{ WVA/LA ${ }^{x}$} \\
\hline & & \multicolumn{4}{|c|}{ Time of measurement (HR) } \\
\hline & & $1030-1100$ & $1330-1400$ & $1030-1100$ & $1330-1400$ \\
\hline 1 & 1.26 & $9.3 \pm 1.3$ & $8.7 \pm 0.8$ & $4.5 \pm 0.05$ & $4.0 \pm 0.05$ \\
\hline 2 & 1.34 & $8.3 \pm 0.7$ & $8.0 \pm 0.7$ & $4.1 \pm 0.07$ & $3.8 \pm 0.06$ \\
\hline
\end{tabular}

${ }^{\mathrm{z}}$ Means are presented $\pm 2 \mathrm{SE}$.

${ }^{y}$ Mean of 15 observations.

${ }^{x}$ Mean of 12 observations.

tify the overall photosynthetic performance of differently trained vineyards and orchards exhibiting peculiar leaf distribution and degree of internal shading. The comparison of light response curves drawn for single leaves and whole trees would also add more information, since, in a very dense canopy the WVA/LA would be expected to rise more slowly with increasing light as compared to a single-leaf response and/or reach saturation at higher irradiance.

\section{Conclusions}

The system configuration reported in our study successfully achieved continuous automated monitoring of whole-vine gas exchange by interfacing various pieces of equipment that are commonly available to researchers in applied plant physiology. Homemade equipment (i.e., psychrometers) also can reduce costs.

To optimize the study of various factors or treatments at the canopy level, considerable time must be devoted to assess whether the plastic enclosure alters the environment around the canopy beyond acceptable limits. In this connection, system calibration vs. an empty chamber is recommended. Varying air flow rates should also be checked to find optimum for $\Delta \mathrm{T}$ and $\Delta \mathrm{CO}_{2}$.

Whole-plant gas exchange systems, as the one described, appear to be powerful tools for detecting, on a whole-canopy level, differences related to shape, leaf distribution and exposure, cropload, biotic and abiotic stresses, or cultural practices. The system can simultaneously monitor several plants, thus allowing treatment replication and is flexible in size of plants that can be measured.

\section{Literature Cited}

American Society of Agricultural Engineers. 1982. Psychrometric data. Amer. Soc. Agr. Eng., St. Joseph, Mich.

Buwalda, J.G., T.G.A. Green, J.S. Meekings, and D.J. Coneybear. 1992. Measurement of canopy gas-exchange of kiwifruit vines using a suite of whole-canopy cuvettes. Environ. Expt. Bot. $32: 425-438$

Corelli-Grappadelli, L. and E. Magnanini. 1993. A whole-tree system for gas-exchange studies. HortScience 28:41-45.

Edson, C.E., G.S. Howell, and J.A. Flore. 1993.
Influence of crop load on photosynthesis and dry matter partitioning of Seyval grapevines. I. Single leaf and whole-vine response pre- and postharvest. Amer. J. Enol. Viticult. 44:139-147.

Garcia, R.L., J.M. Norman, and D.K. McDermitt. 1990. Measurement of canopy gas exchange using an open chamber system. Remote Sensing Rev. 5:141-162.

Heinecke, A.J. and N.F. Childers. 1936. Influence of respiration on the daily rate of photosynthesis of entire apple trees. Proc. Amer. Soc. Hort. Sci. 34:142-146.

Intrieri, C., S. Poni, O. Silvestroni, and I. Filippetti. 1992. Leaf age, leaf position and photosynthesis in potted grapevines. Adv. Hort. Sci. 6:23-27.

Katerjii, N., F.A. Daudet, A. Carbonneau, and N. Ollat. 1994. Etude à l'échelle de la plante entière du fonctionnement hydrique et photosynthétique de la vigne: Comparaison des systèmes de conduite traditionnel et en Lyre. Vitis 33:197203.

Kliewer, W.M. and R.J. Weaver. 1971. Effect of crop level and leaf area on the growth, composition and coloration of Tokay grapes. Amer. J. Enol. Viticult. 22:172-177.

Kriedemann, P.E., W.M. Kliewer, and J.M. Harris. 1970. Leaf age and photosynthesis in Vitis vinifera $\mathrm{L}$. Vitis 9:97-104.

Lakso, A.N., G.B. Mattii, J.P. Nyrop, and S. Denning. 1994. Variazioni di fotosintesi e di crescita dei frutti su piante di melo in relazione alla presenza di acari. Proc. $\Pi^{\circ}$ Giornate Scientifiche S.O.I., S. Benedetto del Tronto, 22-24 giugno:57-58.

Lowe, J. and R. Paul. 1977. An approximating polynomial for computation of saturation vapour pressure. J. Appl. Meteorol. 16:100-103.

McMillen, R.T. 1988. An eddy correlation technique with extended applicability to non-simple terrain. Boundary-Layer Meteorol. 43:231-245.

Mitchell, C.A. 1992. Measurement of photosynthetic gas-exchange in controlled environments. HortScience 27:764-767.

Osborne, W.C. 1977. Fans. 2nd ed. Pergamon Press, Oxford, U.K.

Poni, S., A.N.Lakso, J.R. Turner, and R.E. Melious. 1993. The effects of pre- and post-veraison water stress on growth and physiology of potted Pinot Noir grapevines at varying crop levels. Vitis 32:207-214.

Poni, S., C. Intrieri, and O. Silvestroni. 1994. Interactions of leaf age, fruiting, and exogenous cytokinins in Sangiovese grapevines under nonirrigated conditions. I. Gas-exchange. Amer. J. Enol. Viticult. 45:71-78.

Succi, F., E. Magnanini, and G. Costa. 1990. Interrelations of light, transpiration and $\mathrm{CO}_{2}$ flux exchange in kiwifruit vine. Proc. 3rd Intl.Mtg. on Regulat. of Photosyn. in Fruit Crops. Perugia, Italy, 3-4 Sept. 1990:61-69. 